\title{
Scale ambiguities in material recognition
}

Jacob R. Cheeseman ${ }^{1}$, Roland W. Fleming ${ }^{1,2}$, Filipp Schmidt ${ }^{1}$

${ }^{1}$ Justus Liebig University Giessen

${ }^{2}$ Center for Mind, Brain and Behavior (CMBB)

Author Note:

Jacob R. Cheeseman, Department of Experimental Psychology, Justus Liebig

University Giessen; Roland W. Fleming, Department of Experimental Psychology and Center for Mind, Brain and Behavior (CMBB), Justus Liebig University Giessen and Philipps University of Marburg; Filipp Schmidt, Department of Experimental Psychology, Justus Liebig University Giessen.

This work was funded by the H2020 Marie Skłodowska-Curie Action (H2020MSCA-ITN-2017) 'DyViTo: Dynamics in Vision and Touch'-project number 765121, the Deutsche Forschungsgemeinschaft (DFG, German Research Foundation)-project number 222641018-SFB/TRR 135 TP C1, the European Research Council (ERC) Consolidator Award 'SHAPE'-project number ERC-CoG-2015-682859, and by the Hessisches Ministerium für Wissenschaft und Kunst (HMWK; project 'The Adaptive Mind')

Correspondence concerning this article should be addressed to Jacob R. Cheeseman, Department of Experimental Psychology, Justus Liebig University Giessen, Otto-BehaghelStr. 10F, 35394 Giessen, Germany.

E-mail: jacob.cheeseman@psychol.uni-giessen.de 


\begin{abstract}
Many natural materials have complex, multi-scale structures. Consequently, the inferred identity of a surface can vary with the assumed spatial scale of the scene: a plowed field seen from afar can resemble corduroy seen up close. We investigated this 'materialscale ambiguity' using 87 photographs of diverse materials (e.g., water, sand, stone, metal, wood). Across two experiments, separate groups of participants $(N=72$ adults $)$ provided judgements of the material category depicted in each image, either with or without manipulations of apparent distance (by verbal instructions, or adding objects of familiar size). Our results demonstrate that these manipulations can cause identical images to be assigned to completely different material categories, depending on the assumed scale. Under challenging conditions, therefore, the categorization of materials is susceptible to simple manipulations of apparent distance, revealing a striking example of top-down effects in the interpretation of image features.
\end{abstract}

Keywords: vision; recognition; material perception; spatial perception; scene perception 


\section{Statement of Relevance}

Visually inferring the material properties of objects and surfaces is crucial for many everyday tasks, from selecting food to determining if the ground is slippery. Yet identifying materials from images is challenging due to their enormous variability. Conventional theories of material recognition typically assume a mapping between particular image features and distinct material categories. Here, however, we find that certain images of materials can produce entirely different categorical interpretations depending on the assumed viewing distance. This suggests that material categorization is governed not only by bottom-up interpretations of image features and the physical properties of reflecting surfaces, but also by top-down assumptions about the observer's distance to the surface. Much as familiar objects are associated with canonical sizes, materials are evidently associated with canonical scales, and are recognized through completely different cues at different distances. 


\section{Scale ambiguities in material recognition}

In most circumstances, observers can reliably and efficiently recognize the materials that surfaces are made of (Sharan, Rosenholtz, \& Adelson, 2014). Here, we find, however, that under certain viewing conditions, surfaces made of entirely different materials can produce similar image features, leading to confusions. For example, the mottled surfaces in Fig. 1 are photographs of (a) sea water and (b) marble. When asked to estimate the distances in these images, we are likely to guess that the sea water is much farther from the camera than the marble surface. If we instead imagine that (b) is sea water and (a) is marble, this reverses the difference in assumed distance; that is, sea water only appears this way from a relatively large distance. This 'material-scale ambiguity' suggests that our knowledge of the typical appearance of materials can be used to constrain the range of plausible viewing distances that could produce these images (Fleming, 2014), much like objects of familiar size can indicate depth and distance (Hubbard, Kall, \& Baird, 1989; Konkle \& Oliva, 2011, 2012b, 2012a). Conversely, if material identity and spatial scale are mutually constraining, this also raises the possibility that judgments of material identity may be influenced in a topdown way by the assumed viewing distance to objects and surfaces.

(a)

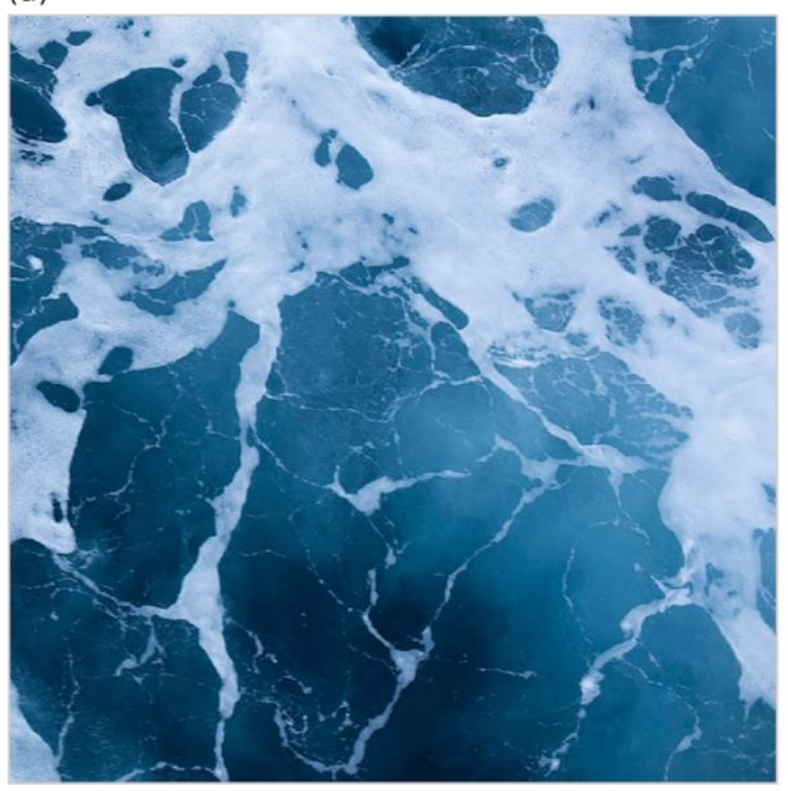

(b)

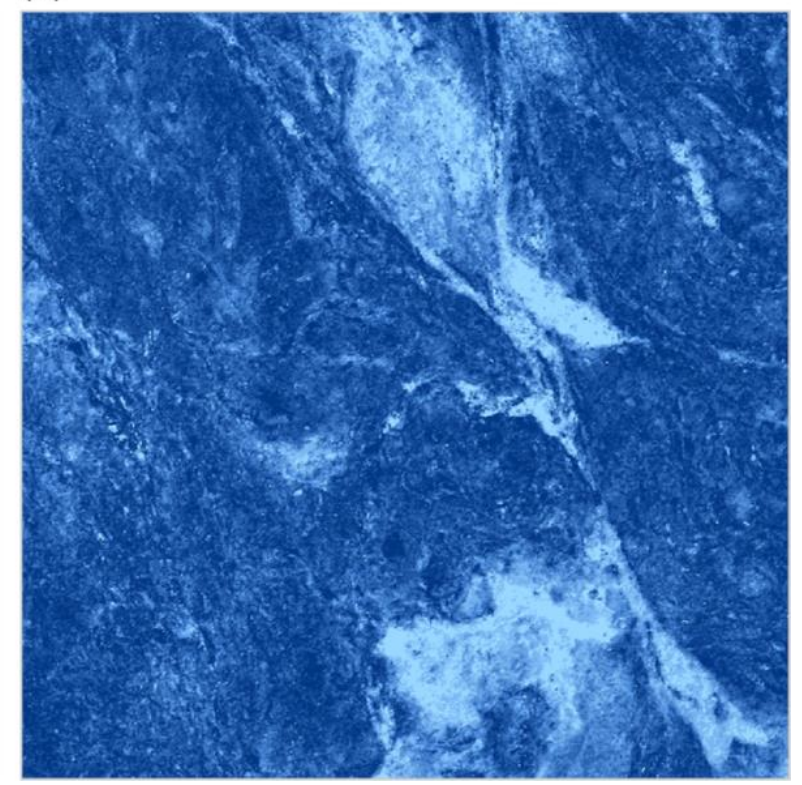


Fig. 1. Demonstration of material and scale dependency. When viewing distance is ambiguous, the material properties depicted in these photographs of (a) sea water and (b) marble are confusable. Note that the relative difference in assumed viewing distance (i.e., the ocean water is farther than the marble surface) reverses if one imagines that the labels are switched.

Material appearance is intimately connected to the spatial scale of geometrical and optical processes at which light interacts with surfaces (Pont \& Koenderink, 2002, 2005). A given material can change in appearance dramatically depending on the viewing distance. Individual water droplets seen close-up are visibly transparent with small punctate highlights, yet when seen en masse from afar, droplets blur together into a white haze. Tiny scratches on the surface of brushed metal appear crisp and mirror-like when viewed through a microscope, yet the ensemble appearance at normal view distances is of a silky, anisotropic gloss (Raymond, Guennebaud, \& Barla, 2016). We reasoned that given the complex, multiscale nature of many materials, image features that emerge at a particular scale or viewing distance can sometimes be deceptive about material properties. For example, anyone who has touched the stems and spikelets of barley knows that they are harsh to touch, yet when seen from afar, a field of barley can have a distinctly 'soft' appearance. This opens the possibility of mis-categorizing materials as a function of the interpreted or assumed viewing distance.

Here, we investigated how contextual information about viewing distance influences material recognition. We did not seek to test the claim that all images of materials suffer from material-scale ambiguities, but rather the more restricted claim that there are certain categories of materials that are confusable when imaged from different viewing distancesand thus that certain images can exhibit this effect. We explicitly set out to find such images and investigate the patterns of confusions they cause. If the same image can be interpreted as completely different material categories depending on the viewer's assumptions about view distance, this has important implications for theories of visual categorization, which typically assume a mapping between particular image features and material categories (Bell, 
Upchurch, Snavely, \& Bala, 2015; Fleming, Wiebel, \& Gegenfurtner, 2013; Sharan, Liu, Rosenholtz, \& Adelson, 2013).

In Experiment 1 we presented participants with (potentially) ambiguous photographs of different materials, and asked them to identify the surface material and estimate the distance between the camera and surface. We reasoned that depending on their interpretation of the surface material, they should judge the viewing distance as relatively small or large. In this way, we could discover not only the typical interpretations of these images, but also the assumed spatial scales associated with these interpretations. We find that indeed, the surfaces depicted in certain images can be identified as completely different materials, depending on whether the observer spontaneously interpreted the surface as seen from near or far. We then sought to test whether we could directly manipulate material recognition by imposing different scale interpretations. In Experiment 2a separate groups of participants were asked to identify the depicted material when instructed to imagine the camera as either very close or very far from the surface plane, and in Experiment $2 \mathrm{~b}$ participants judge modified sets of images that included familiar objects (insect or airplane) to provide contextual information about spatial scale. Our aim was to identify a subset of images for which these manipulations are effective rather than assessing the level of general ambiguity across all images. We hypothesized that if material recognition depends on assumptions about egocentric distance, the pattern of responses in each experiment would depend on the particular instructions or familiar objects provided to participants.

\section{Experiment 1: Unbiased Judgements of Distance and Material}

We first sought to measure baseline judgements of distance and material for our stimulus set in the absence of explicit distance or scale information. Participants were presented with a series of potentially ambiguous photographs and asked to estimate the 
distance between the camera and depicted surface plane, and to identify the material category. The unbiased responses from this experiment served as a baseline of comparison with Experiment 2, in which judgements of distance and material were manipulated with contextual information.

\section{Method}

\section{Participants}

A convenience sample of 24 students ( 14 women, 10 men; $M_{\text {age }}=24.3$ years, $S D_{\text {age }}=$ 3.3 years) participated in the experiment for $8 €$ per hour. All experimental procedures were approved by the Justus Liebig University Giessen Psychology Department Ethics Board and conformed with the guidelines of the American Psychological Association (Version 2017) and the Declaration of Helsinki (Version 2013). Only one previous study (Wiebel, Valsecchi, \& Gegenfurtner, 2013; $N=18$ ) has estimated material categorization accuracy under conditions similar to the current study, that is, with unlimited stimulus presentation time and no instruction to respond rapidly. All of the participants in that study achieved categorization accuracies $(M d n=87 \%)$ that exceeded chance-level accuracy $(25 \%)$. According to the $95 \%$ confidence interval (CI) obtained from a one sample binomial test, the probability that the observed median categorization accuracy exceeds chance-level is between $85 \%$ and $100 \%, p$ $<0.001, g=0.62$. Although this indicates that material categorization is quite robust under normal conditions, given that our stimuli were selected for ambiguity, a larger sample $(N=$ 24) was preferred for Experiment 1.

\section{Stimuli}

Potentially ambiguous photographs were collected from various internet sources. The selection criteria were as follows: (i) they did not contain objects, but clearly depicted a surface or scene, (ii) they were determined by the experimenter to be potentially ambiguous 
in the sense that at least two distinct material categories could describe the surface or scene, and (iii) these material categories could be associated with different spatial scales. Three observers (including two authors) screened hundreds of images on the internet and selected those that met the criteria, resulting in 87 images (Fig. 2a). Ground truth category labels and copyright information for these images are listed in the Supplemental Material (Fig. S1 and Table S1). Ground truth category labels for these images were determined by the original publisher. Images for which print copyright permissions could not be obtained are here replaced by synthetic textures derived from the originals (see Risser, 2020). All images were rescaled to $600 \times 600$ pixels. 
(a)

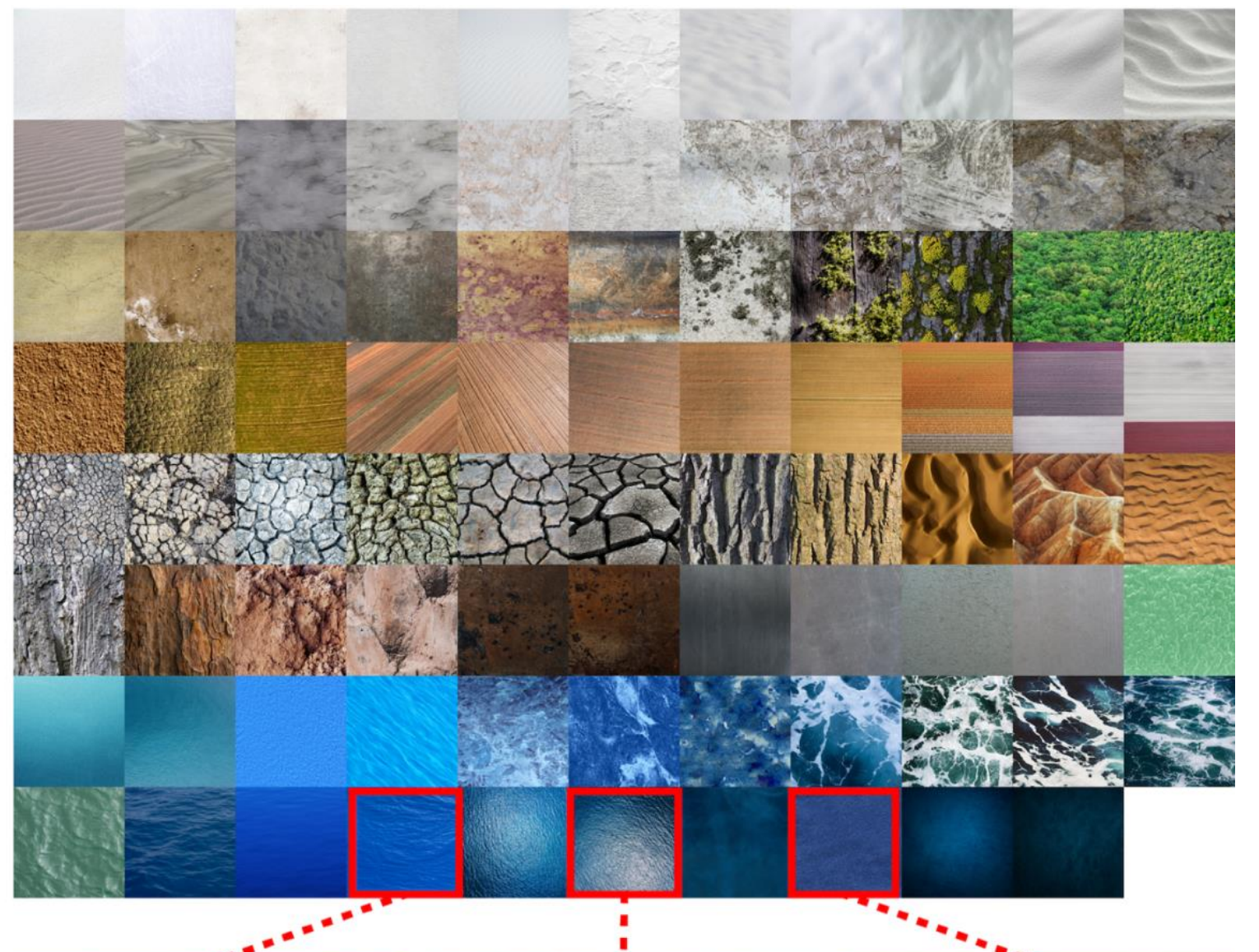

(b)

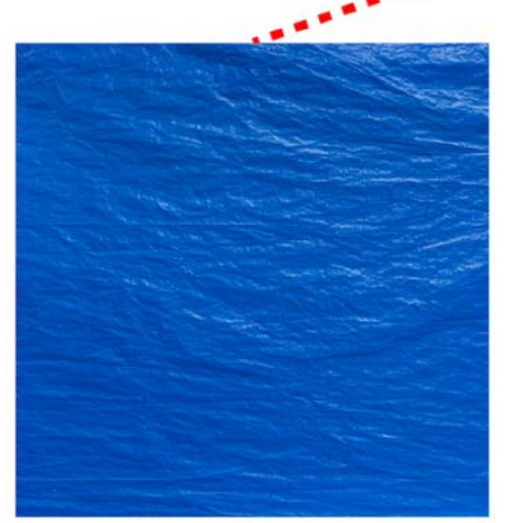

Plastic

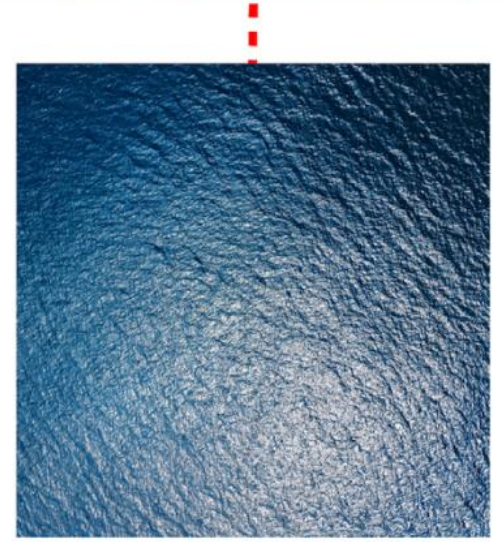

Water

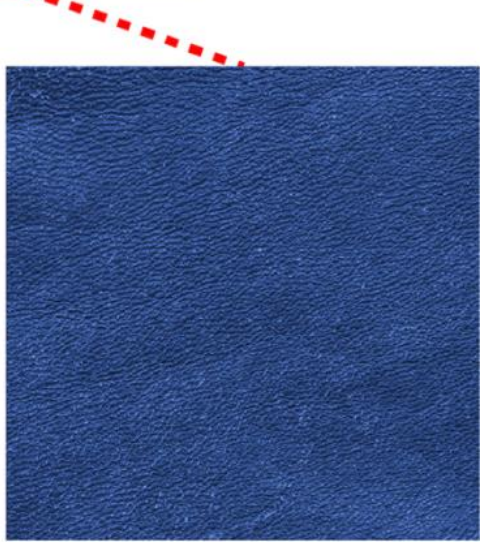

Leather

Fig. 2. Stimuli for Experiments 1 and 2. The full set of 87 images (a) is arranged by approximate visual similarity. Similar image features (e.g., color, texture) can arise from different material categories (b), especially when spatial scale is ambiguous.

\section{Procedure}

On each trial, participants viewed a single, randomly-selected image and judged the depicted material, followed by the distance between the camera and surface plane. Different kinds of responses were collected in separate blocks of trials. In the first block (free- 
response), participants described the surface material as precisely as possible by typing a written response. In the second block (multiple-choice), participants identified surface materials by selecting one category from 26 options (see Multiple Choice Instructions in the Supplemental Material). Participants also provided a confidence rating (from 1 to 7 ) for judgements of material. In the third block (distance estimation), participants estimated the distance between the camera and surface plane by selecting an appropriate unit of measurement (micrometers, millimeters, centimeters, meters, or kilometers) and metric value (e.g., $8 \mathrm{~cm}$, or $1 \mathrm{~km}$ ). English translations of the original German instructions for all experiments are provided in the Supplemental Material.

\section{Results}

Our first main finding is that overall, participants were quite good at identifying the correct categories of materials. All participants achieved categorization accuracies $(M d n=$ $33 \%$ ) that exceeded chance-level accuracy (4\%). According to the $95 \%$ confidence interval (CI) obtained from a one sample binomial test, the probability that the observed median categorization accuracy exceeds chance-level is between $96 \%$ and $100 \%, p<0.001, g=0.29$. Additionally, there was a strong rank correlation between the frequency of common terms represented in the free-response and multiple-choice data $\left(r_{s}=0.79, p<0.001\right.$; see FreeResponse Transformations in the Supplemental Material for details). These findings show that although the images were selected for their ambiguity, participants' responses were systematic and consistent across tasks. Thus, deviations from ground truth were likely purposeful. We next sought to answer whether such errors were systematically related to the assumed scale (or distance) of the scene.

To assess this, we divided the multiple-choice responses for each image into two distance groups: (i) responses from participants who selected distance units indicating a 
relatively small scale (near group: micrometer, millimeter, or centimeter), and (ii) responses from participants who selected distance units that indicated a relatively large scale (far group: meter or kilometer). Material category confusions can occur in two directions (near $\rightarrow$ far vs. far $\rightarrow$ near). Some confusions between categories are likely to be distance-independent simply because the materials are similar in appearance (e.g., confusing stone and concrete irrespective of distance), and will therefore tend to be symmetrical in each direction. Instead, we focus on asymmetrical patterns of confusion that reflect a distance-dependent material ambiguity. That is, are there images that are assigned to one material (e.g., bark) when seen as close-up, but which are assigned to another material (e.g., stone) when seen as far away, but not vice versa?

The relative frequency of directional category confusions is indicated by shaded lines in Fig. 3a. The observed asymmetry between the pattern of blue lines (near $\rightarrow$ far confusions) and red lines (far $\rightarrow$ near confusions) illustrates that material category confusions vary systematically with assumed viewing distance. For clarity, cases where the two distance groups selected the same category are omitted, and only categories corresponding to ground truth image labels are shown (for details, see Relative Frequencies in the Supplemental Material).

Fig. $3 \mathrm{~b}$ displays the same relative frequencies (here normalized between 0 and 1) plotted as cells in a matrix, with responses of the near and far groups represented on separate axes. Category confusions that occur in the near $\rightarrow$ far direction are located in the lower triangle of the matrix (blue-shaded cells), and confusions in the far $\rightarrow$ near direction are located in the upper triangle (red-shaded cells). Instances where the two distance groups selected the same category are plotted on the diagonal of the matrix (purple-shaded cells). Directional asymmetries can be compared by examining category pairs on opposite sides of the diagonal. For example, we find that surfaces identified as bark at smaller apparent 
distances tend to be confused with stone at larger apparent distances (frequency $=0.4$; blue square in Fig 3b). However, the reverse is not true: when a surface is seen as bark at larger apparent distances, it is less often confused with stone at smaller apparent distances (frequency $=0.1$; yellow square in Fig $3 b$ ). Fig $3 c$ shows one such image, and for comparison Fig $3 \mathrm{~d}$ shows an image with a distance-dependent ambiguity in the opposite direction (water seen from far looks like plastic seen from near).

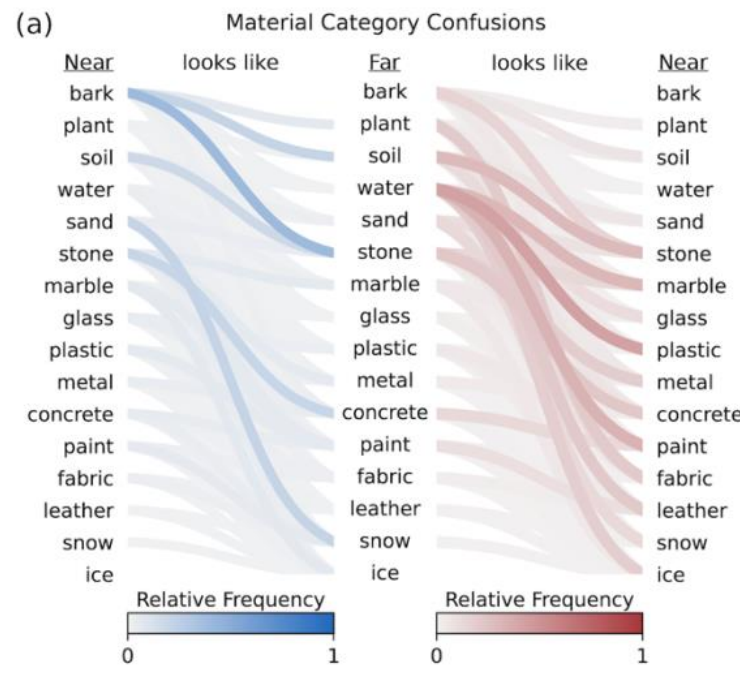

(c)

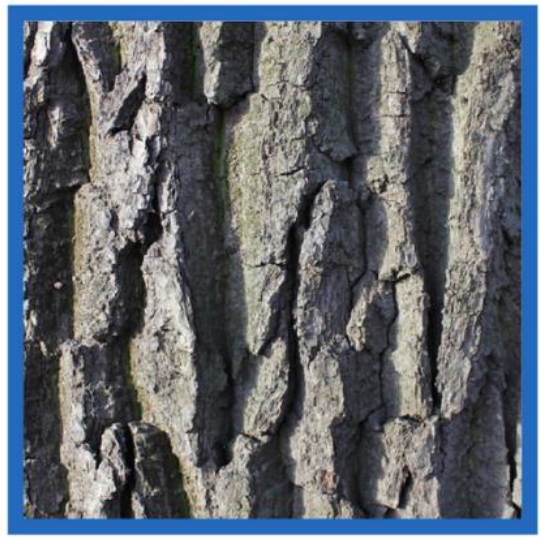

Bark (Near) looks like Stone (Far) (b)

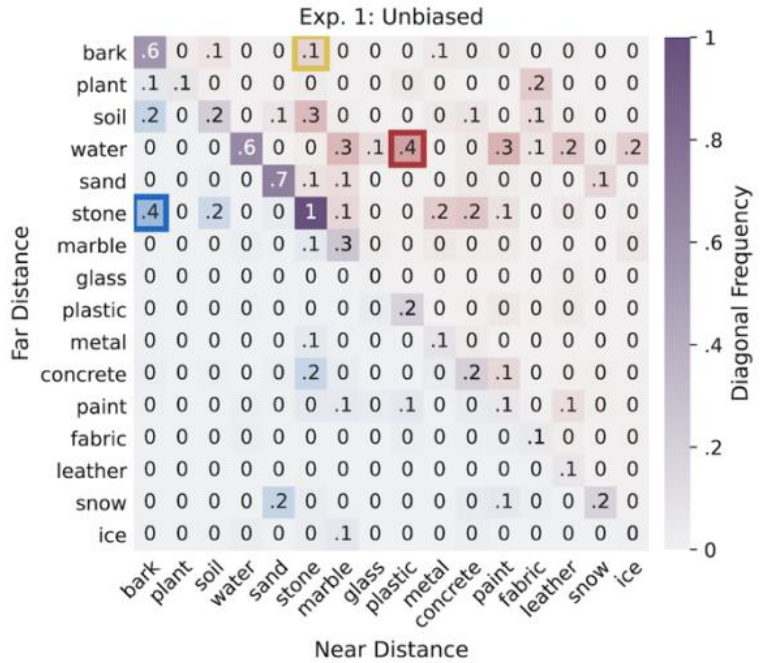

(d)

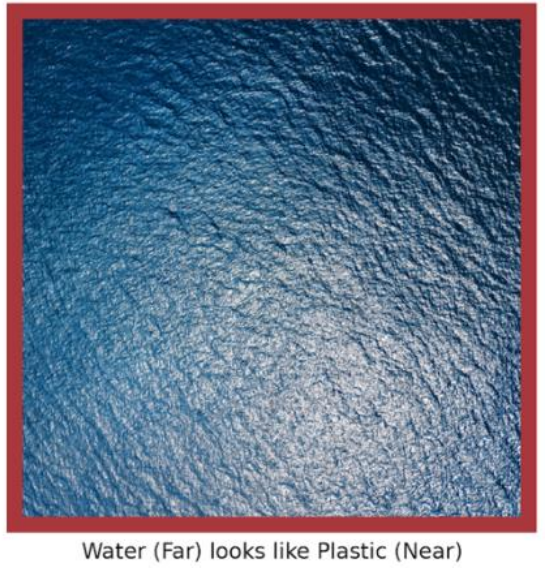

Fig. 3. Material category confusions exhibiting material-scale ambiguity. The relative frequency of confusions between pairs of categories is indicated by shaded connecting lines in (a). Categories selected with smaller distance units (micrometer, millimeter, or centimeter) are defined as near, while categories selected with larger distance units (meter or kilometer) are defined as far. Confusions in the near $\rightarrow$ far direction (blue-shaded lines) differ systematically from confusions in the far $\rightarrow$ near direction (red-shaded lines). The same asymmetry can be seen when (normalized) relative frequencies are plotted in a matrix format (b), with distance-dependent responses on separate axes. Category pairs above and below the negative diagonal indicate the relative frequency of distance-dependent confusions; distance- 
independent responses are shown on the diagonal. The top-ranked images for the most frequent near $\rightarrow$ far confusion (c) and the most frequent far $\rightarrow$ near confusion (d) illustrate different material-scale ambiguities.

The matrix representation of category confusions also provided a simple way to measure the overall magnitude of material-scale ambiguity for this set of images. This is accomplished by calculating the Root Mean Squared Error (RMSE) between corresponding frequencies in the lower and upper triangles of the matrix (e.g., blue cells vs. red cells in Fig 3b, excluding the diagonal). This metric, which we call the Material-Scale Ambiguity (MSA), represents the amount of directional asymmetry in category confusions. MSA can also be calculated for individual images, which allows us to identify the subset of images that were particularly effective in producing distance-dependent category confusions. MSA for individual images was then compared with asymmetries that occur by chance, by calculating the mean MSA across 1000 random permutations of the distance units associated with each response (i.e., category confusions are shuffled in each direction). Fig. 4a shows images ranked by MSA, with the corresponding chance-level MSA drawn in lilac. Sixty-one (out of 87) images produced MSA values above chance. Fig. 4b shows that the mean MSA across all images (teal; $M=1.4,95 \%$ CI $[1.3,1.51]$ ) was significantly greater than chance-level MSA (lilac; $M=0.95,95 \%$ CI $[0.88,1.02]$; Wilcoxon $T=850, p<0.001, d=0.52$ ). This result indicates that, as a whole, the image set produces systematic distance-dependent category confusions, but also that there is a subset of images that are particularly ambiguous in this way. For example, the image shown in Fig. 4c was reliably seen as bark regardless of apparent distance, whereas the image shown in Fig. 4d produced confusions between plant (seen from a relatively large distance) and carpet (seen from a relatively small distance). The material categories associated with relatively small or large assumed distances are shown in word clouds below each image, where category frequency is indicated by word size. 
(a)

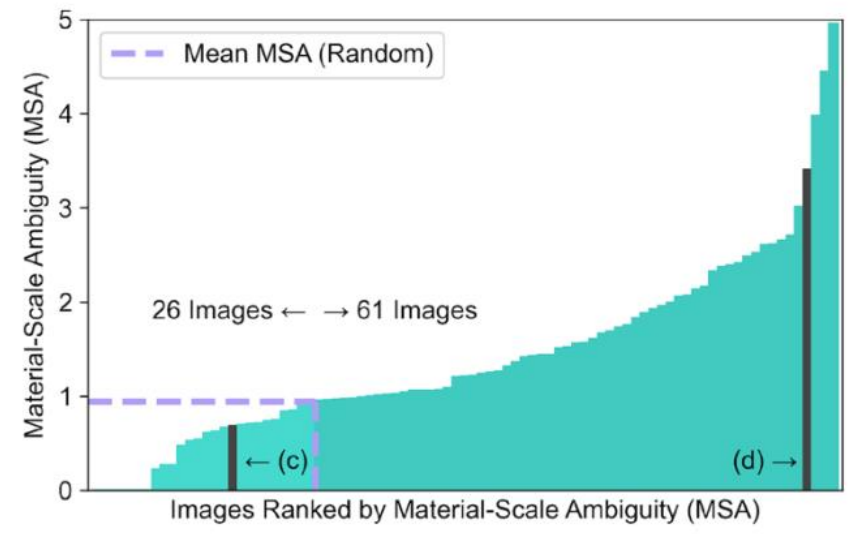

(b)

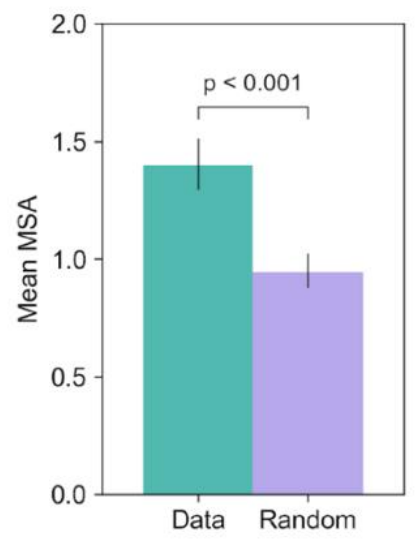

(c)

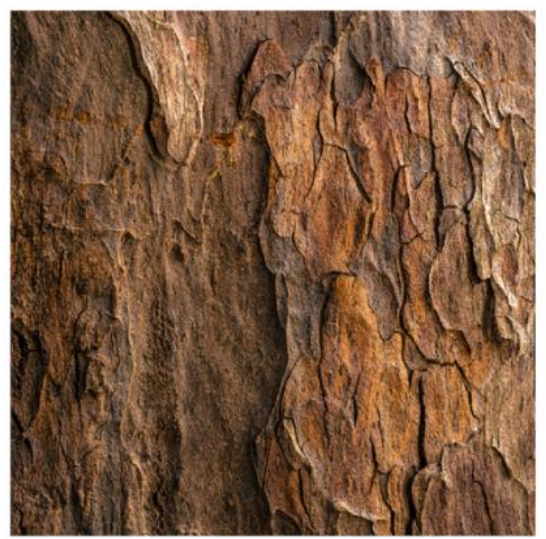

bark

near

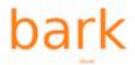

far

(d)

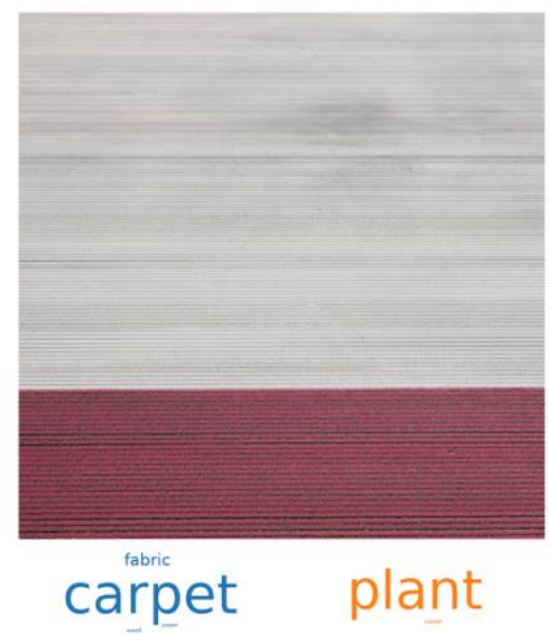

Fig. 4. Material-Scale Ambiguity (MSA) calculated for individual images. Images can be ranked by MSA (a) along with the mean MSA resulting from random permutation of distance units drawn in lilac. The mean MSA across images (b) for the original responses (teal) and random permutation of distance units (lilac). A Wilcoxon signed-rank test indicated that the observed difference in MSA is significant. Error bars represent 95\% confidence intervals. An image with low MSA (c) is reliably seen as one material (bark) regardless of assumed distance. Word clouds of responses (size weighted by frequency) associated with different distance assumptions are shown below. An image with high MSA (d) is reliably seen as different materials (carpet vs. plant) depending on the assumed distance (far vs. near).

Taken together, the results of this experiment show that, without contextual information to specify viewing distance, certain images exhibit a striking distance-dependent ambiguity about the category of material. The material category labels assigned to these images covary with whether the surfaces are interpreted as near to or far from the camera. To test whether the assumed viewing distance plays a causal role in driving this effect, we next sought to directly manipulate assumed viewing distance. 


\section{Experiment 2: Biased Judgements of Distance and Material}

To test whether manipulating assumed viewing distance influences judgements of material, we presented the same stimuli to four additional groups of naïve participants. Assumed viewing distance was manipulated by instructing each group to imagine a small or large distance to the surface plane (Experiment 2A), or by presenting modified images that featured two different familiar objects to indicate different spatial scales (Experiment 2B).

\section{Method}

\section{Participants}

Four groups of 12 students (48 total) participated in the experiment. Group 1 (9 women, 3 men; $M_{\text {age }}=24.5$ years, $S D_{\text {age }}=3.01$ years $)$ and Group $2\left(9\right.$ women, 3 men; $M_{\text {age }}=$ 22.5 years, $S D_{\text {age }}=3.64$ years) participated in Experiment $2 \mathrm{~A}$. Group 3 ( 4 males and 8 females; $M_{a g e}=24.5$ years, $S D_{a g e}=4.01$ years $)$ and Group 4 ( 4 males and 8 females; $M_{a g e}=$ 22.83 years, $S D_{\text {age }}=2.37$ years) participated in Experiment $2 \mathrm{~B}$.

\section{Stimuli}

The same set of images presented in Experiment 1 was shown to each group of participants in Experiment 2A. For Experiment 2B, we created two sets of images from the original set, each of which featured a large or small familiar object (3D models of an airplane or a hornet) that provided contextual information about the spatial scale of the scene. The objects were digitally inserted into the images with 3D modelling software that simulated lighting direction and cast shadows (for details, see Experiment 2 Stimuli in the Supplemental Material). 


\section{Procedure}

Randomly selected images from the corresponding set were presented to participants in the same viewing conditions as in Experiment 1. For each image, participants identified the depicted surface material (free-response and multiple-choice, in separate blocks of trials) and indicated their confidence (1-7). In Experiment 2A, Group 1 was instructed to imagine that the camera was "very far" from the surface plane, and Group 2 was instructed to imagine that the camera was "very near" to the surface plane. These instructions were intended to ensure that the imagined distance range could vary with the interpretations for each image. In Experiment 2B, Group 2 was presented with images that featured a large familiar object (airplane), and Group 3 was presented with images that featured a small familiar object (hornet).

\section{Results}

Material-Scale Ambiguity (MSA) was calculated separately for Experiment 2A and Experiment 2B. To test whether the observed asymmetries in category confusions were statistically significant, we compared them to chance-level MSA calculated from 1000 random permutations of group membership in each experiment. Images ranked by MSA for all experiments are shown in Fig. 5a, along with chance-level MSA in lilac. Fig. 5b shows the mean MSA across images for the original responses (teal) and chance-level MSA (lilac) separately for all experiments. In Experiment 2A, values of MSA for the original responses $(\mathrm{M}=1.48,95 \% \mathrm{CI}[1.37,1.6])$ were significantly greater than chance-level MSA $(M=1.15$, 95\% CI [1.07, 1.25]; Wilcoxon $T=799, p<0.001, d=0.49)$. Magnitudes of MSA produced by manipulation of assumed viewing distance with instructions (Experiment 2A) are therefore similar to those produced by unbiased judgements of material and distance (Experiment 1). Although familiar objects produced above-chance MSA in 46 images 
(Experiment 2B), this manipulation was less effective overall (Wilcoxon $T=1812, p=0.80$, $d=0.07)$. Interestingly, confidence ratings in Experiment $2 \mathrm{~B}(M=4.8, S D=0.71)$ were significantly lower than those in Experiment 2A $(M=4.98, S D=0.5$; Mann-Whitney $U$ $=192.5, p=0.025, d=0.25)$. It is also noteworthy that in general, the same images produced high MSA across experiments. Values of MSA in Experiment 1 are significantly correlated in rank with those obtained in Experiment $2 \mathrm{~A}\left(r_{s}=.43, p<.001\right)$ and Experiment $2 \mathrm{~B}\left(r_{s}=.28, p\right.$ $=.009)$; the rank correlation between Experiment 2A and 2B was not significant. These results suggest that, compared to instructions, the effectiveness of familiar objects is more dependent on whether the objects appear plausible within a given scene.

(a)

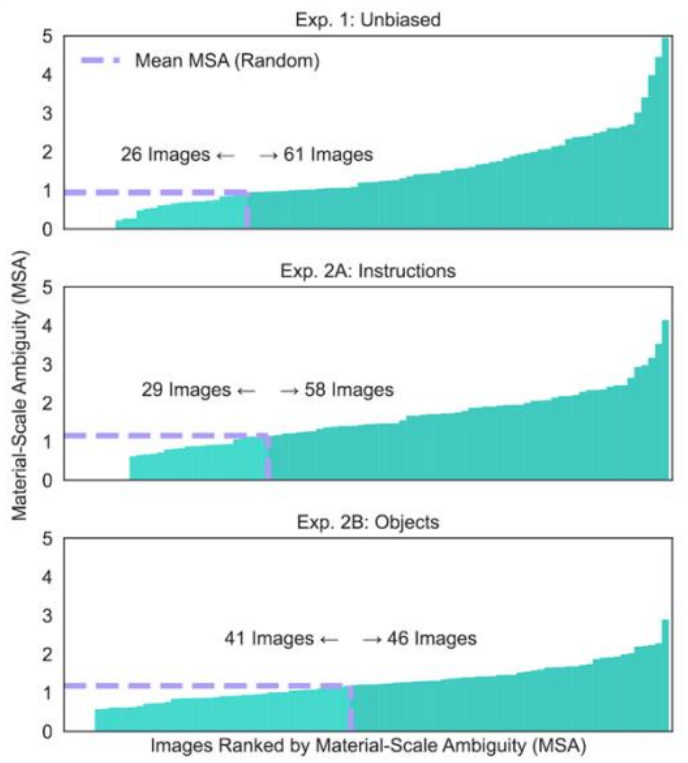

(b)

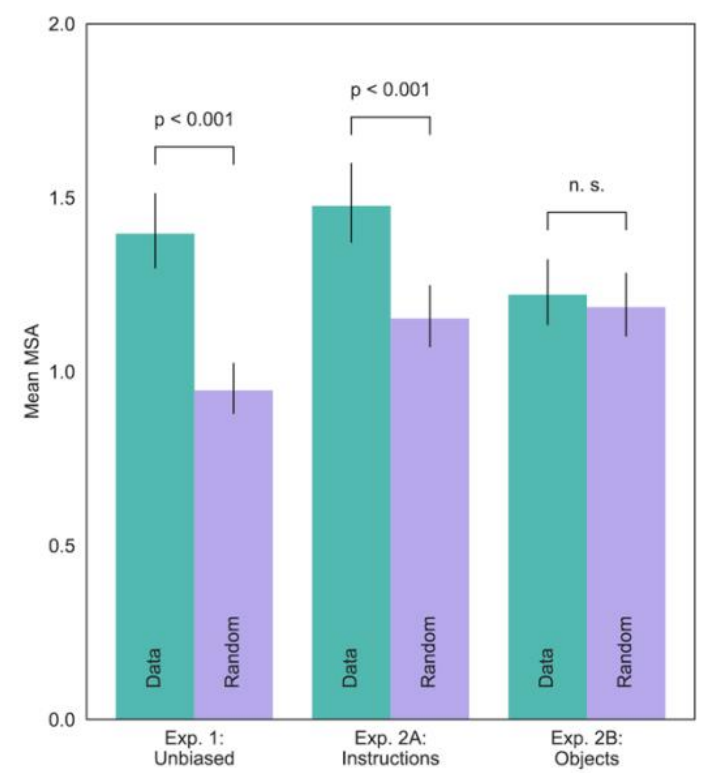

Fig. 5. Material-Scale Ambiguity (MSA) calculated separately for each experiment. (a) Images ranked by MSA. The mean MSA resulting from random permutation of distance units is drawn in orange. (b) Mean MSA across images for the original responses is shown in blue, while mean MSA obtained from random permutation of distance units (Experiment 1) or group membership (Experiment 2) is shown in orange. The observed difference in MSA is significant for Experiments 1 (unbiased) and 2A (instructions). Error bars represent 95\% confidence intervals. 
Ranking our images by the mean MSA across experiments provides the clearest summary of our findings (Fig. 6). Images with low MSA (bottom row) are immune to manipulations of viewing distance. Yet, when MSA is high (top row), the assumed distance between the camera and the surface plane determines which categories are selected.

(a)
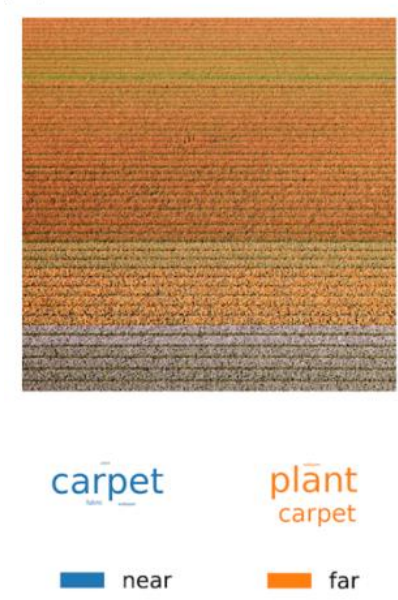

(b)

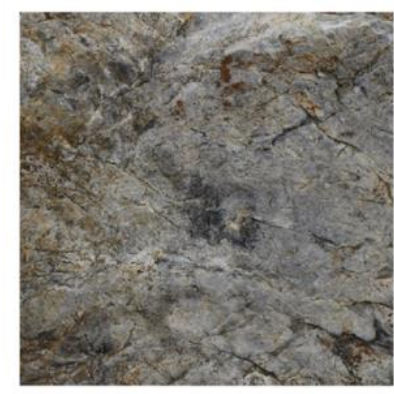

stōne

near
Highest Material-Scale Ambiguity

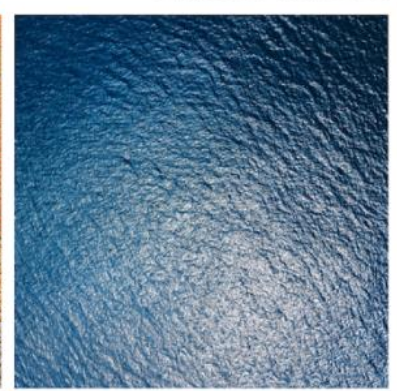

plastic

paint

water
leather

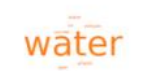

wăter

marble

sāind

carperpet

carpet
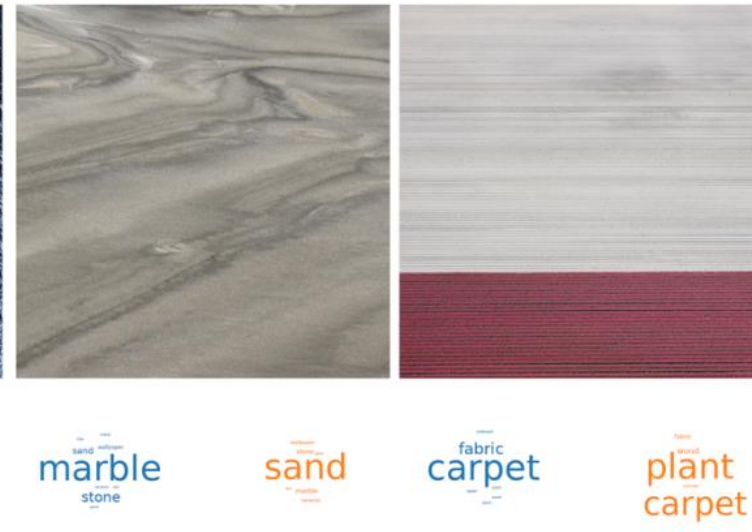

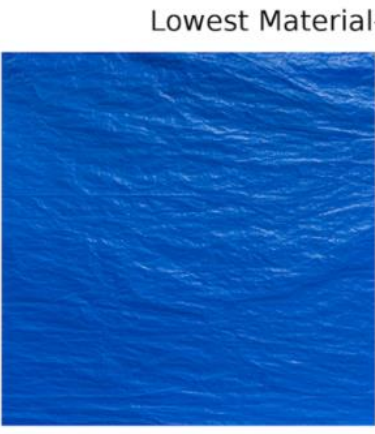

plastic

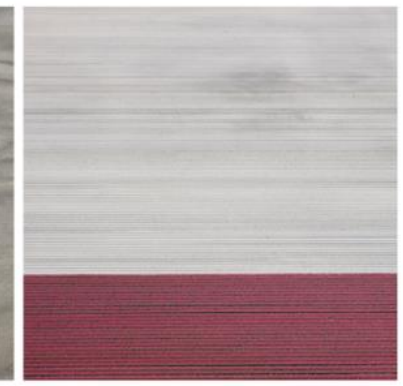

Lowest Material-Scale Ambiguity
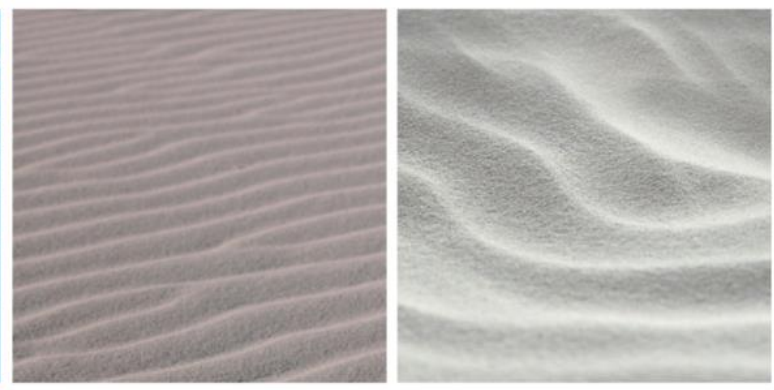

sānd

sand

Fig. 6. Images from our set with the highest (a) and lowest (b) MSA across experiments. Word clouds below each image show the distinct material categories (size weighted by frequency) selected by participants who assume relatively small or large distances to the surface plane (blue and orange data, respectively).

\section{General Discussion}

Surface material appearance is characterized by complex physical and optical structures at multiple spatial scales. As materials can have strikingly different appearances at different viewing distances, we reasoned that for certain images, the appearance of a material 
at one distance could potentially resemble the appearance of another material at a different distance, leading to different category assignments. Here, we have established that such images exist and shown how the assumed view distance can radically alter how they are interpreted.

In Experiment 1, we found spontaneous confusions about material category cooccurring with substantial differences in the assumed viewing distance. In Experiment 2, we showed that both instructions and the insertion of familiar objects indicating different physical scales can alter material classification in a subset of images. An important caveat is that our conclusions are necessarily dependent on the images in our stimulus set. Our goal was to identify specific images that are susceptible to such manipulations, rather than to draw conclusions about the frequency of such ambiguities across all natural images. Although we rarely make such confusions about material categories in everyday life, the very existence of such images demonstrates a substantial top-down effect in visual recognition, in which context can radically alter the interpretation of identical images.

Our finding that distance assumptions can bias material categorization supports the notion that materials have canonical scales that constrain diagnostic image cues. Much as certain viewpoints and sizes of objects can be considered canonical (Konkle \& Oliva, 2011; Palmer, Rosch, \& Chase, 1981), image cues associated with particular material categories may be more likely to arise at typical viewing distances (Fleming, 2014). Conversely, when a material is viewed from an atypical distance (e.g., in macro or aerial photography; see De Giuli, 2018), the cues may resemble other materials, resulting in material-scale ambiguities. To date, theories of material perception and appearance have tended to ignore their scalespecificity; future work on material recognition should consider how material appearance and relevant image features vary with distance. 


\section{Image cues and material-scale ambiguity}

Which image characteristics cause material-scale ambiguity? The images in Figure 6 hint at some compositional elements that might contribute. When the camera is (approximately) perpendicular to the surface plane, this limits depth cues (e.g., texture and optical blur gradients; Sedgwick, 2005; Vishwanath, 2010), increasing ambiguity. The absence of visible object boundaries likely also plays an important role, although many such unbounded texture images are unambiguous (Fleming et al., 2013; Wiebel et al., 2013).

It is therefore unlikely that there is a single image characteristic that determines whether a given image exhibits material-scale ambiguity. Yet, for a given material category, there may be certain cues that are particularly important. For example, Sawayama, Nishida, and Shinya (2017) have shown that participants can judge the fineness of fibrous textures (like human hair), even when individual texture elements are smaller than the resolution of the imaging system. The main cue driving the super-resolution judgments in their displays was contrast: lower contrast patterns appeared to contain finer elements due to averaging of texture elements within each pixel. If the visual system relies on such inferences beyond the image data to infer specific material properties, it is necessarily open to scale-related ambiguities. Relatedly, Ritchie and van Buren (2020) note a curious motif in the work of René Magritte (e.g., The Happy Donor, 1966), in which the interpretation of image regions as materials or scenes is determined by figure-ground assignment. The imagined reversal of figure and ground in these images produces a compelling impression of looking through an interior window onto an exterior scene, or alternatively, looking at a textured foreground surface which stands in front of a relatively uniform background. The interpretation of Magritte's motif, as with the images used in our experiments, relies on the 'beholders share' rather than image statistics per se (Gombrich, 1961). 


\section{Limitations and Future Directions}

The conclusions we can draw from this study are necessarily limited to specific images that exhibit material-scale ambiguity. Further investigation of this effect — and how it relates to material perception in general—will require innovations that lower the difficulty of creating or discovering such images. One approach to probing the characteristics of such images is to blend the statistics of different images that produce the same kind of materialscale ambiguity. For example, Fig. 7a displays an image of bark that was often confused with stone, together with two synthetic 'lookalikes' generated by a deep convolutional neural network used for image style transfer (Gatys, Ecker, \& Bethge, 2016). In this example, the 'styles' are other images of bark that were often confused with stone (i.e., the ground truth category is the same in the style and transfer images). A variation of this approach could involve mixing images that differ in ground truth category, but which produce the same kind of material-scale ambiguity (e.g., the images of water and leather in Fig. 7b). An intriguing alternative approach would be to ask artists to create ambiguous images and analyze the techniques they use to depict material appearance (Di Cicco, van Zuijlen, Wijntjes, \& Pont, 2021; Lin, Van Zuijlen, Wijntjes, Pont, \& Bala, 2021). Such methods may make it possible to identify key features that alter perceived scale or material appearance. 
(a)

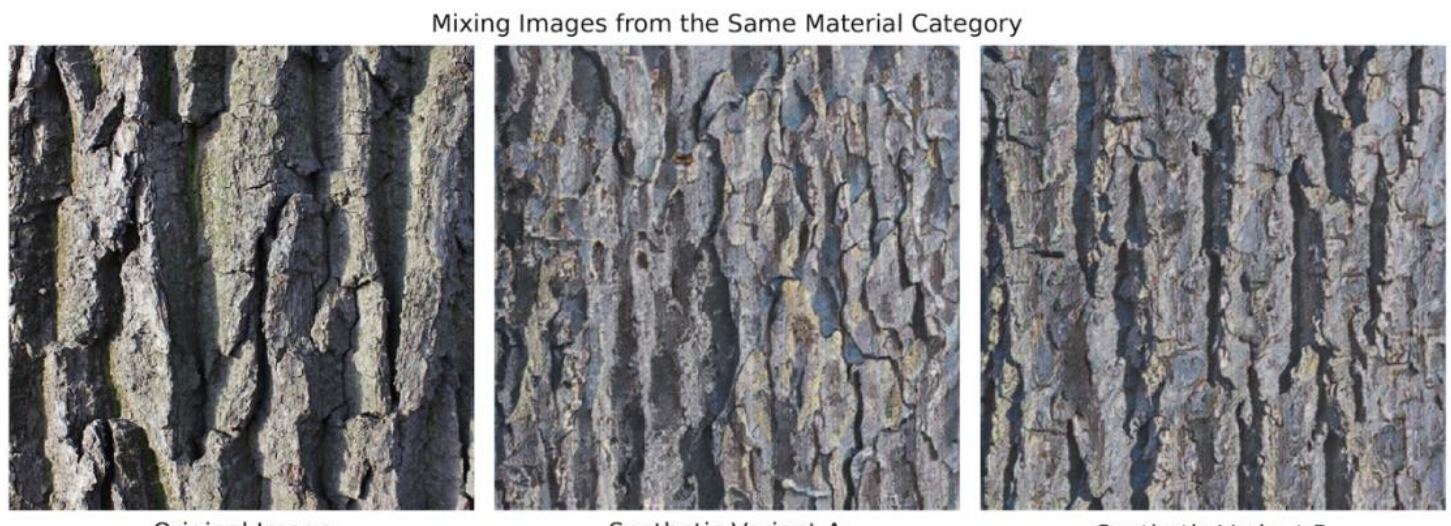

Original Image

Synthetic Variant A

Synthetic Variant B

(b)

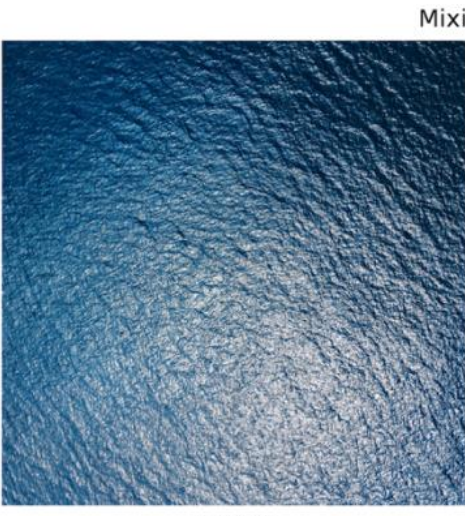

Water

Mixing Images from Different Material Categories

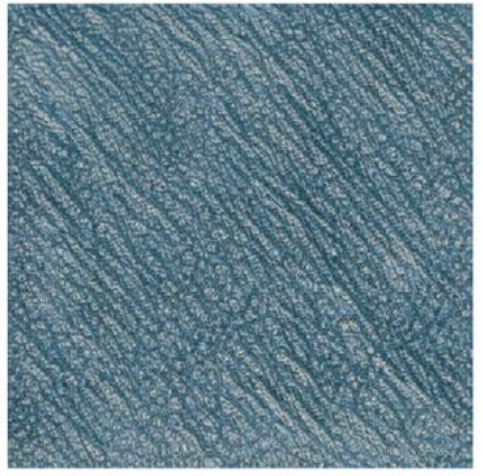

Water-Leather Mixture

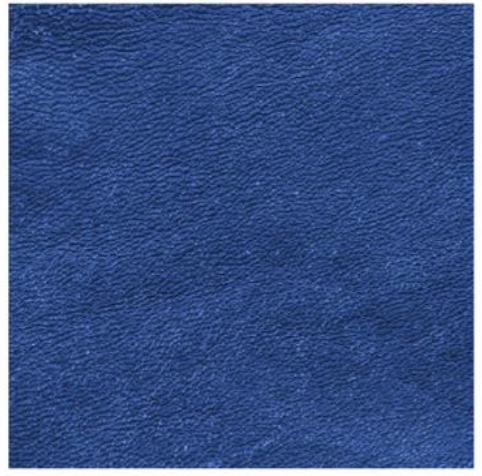

Leather

Fig. 7. Material ambiguity might be created with the aid of deep neural networks. (a) An original image from our set can be modified to mimic the image statistics of other exemplars from the same material category. (b) Original images from different material categories (water and leather) can be used to create a synthetic image that inherits the statistics of both images. These techniques could be used to aid the discovery or invention of images that possess specific kinds of material ambiguity.

\section{Conclusions}

Our findings reveal a novel top-down effect in visual categorization. We find that certain images can appear to depict two or more completely different categories of material, depending on the apparent or implied viewing distance — a material-scale ambiguity. Future research focusing on automatically finding and synthesizing images with scale-dependent material ambiguity could inform theories of material perception, and more broadly, how image statistics relate to image interpretation. 


\section{Author Contributions}

J. R. Cheeseman and F. Schmidt developed the study concept and designed the experiments. J. R. Cheeseman programmed and ran the experiments. J. R. Cheeseman performed the data analysis and interpretation under the supervision of R. W. Fleming and F. Schmidt. J. R. Cheeseman drafted the manuscript, and R. W. Fleming and F. Schmidt provided critical revisions. All authors approved the final version of the manuscript for submission.

\section{Declaration of Conflicting Interests}

The author(s) declare that there were no conflicts of interest with respect to the authorship of this article.

\section{Funding}

This work was funded by the H2020 Marie Skłodowska-Curie Action (H2020-MSCA-ITN2017) 'DyViTo: Dynamics in Vision and Touch'-project number 765121, the Deutsche Forschungsgemeinschaft (DFG, German Research Foundation)-project number 222641018SFB/TRR 135 TP C1, the European Research Council (ERC) Consolidator Award 'SHAPE'project number ERC-CoG-2015-682859, and by the Hessisches Ministerium für Wissenschaft und Kunst (HMWK; project 'The Adaptive Mind')

\section{Open Practices}

Neither of the experiments reported in this article were formally preregistered. De-identified data for both experiments are posted at:

https://osf.io/ukq95/?view_only=b820c135262c48ae8698ddd877063255. Stimulus images for both experiments are provided, except when copyright permission could not be obtained. 


\section{ORCID iD}

Jacob R. Cheeseman: https://orcid.org/0000-0001-9306-537X

Roland W. Fleming: https://orcid.org/0000-0001-5033-5069

Filipp Schmidt: https://orcid.org/0000-0001-8023-7304

\section{Acknowledgments}

We thank Jasmin Kleis and Saskia Lea Honnefeller for help in data collection, Thomas

Heinrich Schmidt for helpful suggestions on data analysis, and Arash Akbarinia for help with texture synthesis. An early version of this work was presented at the $42^{\text {nd }}$ European Conference on Visual Perception (August 2019, Leuven). 


\section{References}

42nd European Conference on Visual Perception (ECVP) 2019 Leuven. (2019). Perception, 48(2_suppl), 1-236. https://doi.org/10.1177/0301006619863862

Bell, S., Upchurch, P., Snavely, N., \& Bala, K. (2015). Material recognition in the wild with the materials in context database. In The IEEE Conference on Computer Vision and Pattern Recognition (CVPR).

De Giuli, R. (2018). ICN T1. Retrieved October 30, 2020, from https://www.terracollage.com/icn-t1

Di Cicco, F., van Zuijlen, M. J. P., Wijntjes, M. W. A., \& Pont, S. C. (2021). Soft like velvet and shiny like satin: Perceptual material signatures of fabrics depicted in 17 th century paintings. Journal of Vision, 21(5), 10. https://doi.org/10.1167/jov.21.5.10

Fleming, R. W. (2014). Visual perception of materials and their properties. Vision Research, 94, 62-75. https://doi.org/10.1016/j.visres.2013.11.004

Fleming, R. W., Wiebel, C., \& Gegenfurtner, K. (2013). Perceptual qualities and material classes. Journal of Vision, 13(8), 9. https://doi.org/10.1167/13.8.9

Gatys, L. A., Ecker, A. S., \& Bethge, M. (2016). Image style transfer using convolutional neural networks. In Proceedings of the IEEE Conference on Computer Vision and Pattern Recognition (CVPR). 
Gombrich, E. H. (1961). Art and illusion: A study in the psychology of pictorial representation. Princeton, NJ: Princeton University Press.

Hubbard, T. L., Kall, D., \& Baird, J. C. (1989). Imagery, memory, and size-distance invariance. Memory \& Cognition, 17(1), 87-94. https://doi.org/10.3758/BF03199560

Konkle, T., \& Oliva, A. (2011). Canonical visual size for real-world objects. Journal of Experimental Psychology: Human Perception and Performance. https://doi.org/10.1037/a0020413

Konkle, T., \& Oliva, A. (2012a). A familiar-size Stroop effect: Real-world size is an automatic property of object representation. Journal of Experimental Psychology: Human Perception and Performance. https://doi.org/10.1037/a0028294

Konkle, T., \& Oliva, A. (2012b). A real-world size organization of object responses in occipitotemporal cortex. Neuron, 74(6), 1114-1124. https://doi.org/10.1016/j.neuron.2012.04.036

Lin, H., Van Zuijlen, M., Wijntjes, M. W. A., Pont, S. C., \& Bala, K. (2021). Insights from a large-scale database of material depictions in paintings. In A. Del Bimbo, R. Cucchiara, S. Sclaroff, G. M. Farinella, T. Mei, M. Bertini, ... R. Vezzani (Eds.), International Conference on Pattern Recognition (pp. 531-545). Springer International Publishing. https://doi.org/10.1007/978-3-030-68796-0_38

Palmer, S. E., Rosch, E., \& Chase, P. (1981). Canonical perspective and the perception of objects. In J. Long \& A. Baddeley (Eds.), Attention and performance IX (pp. 135-151). 
Hillsdale, NJ: Lawrence Erlbaum Associates.

Pont, S. C., \& Koenderink, J. J. (2002). Bidirectional reflectance distribution function of specular surfaces with hemispherical pits. Journal of the Optical Society of America A, 19(12), 2456-2466. https://doi.org/10.1364/JOSAA.19.002456

Pont, S. C., \& Koenderink, J. J. (2005). Bidirectional texture contrast function. International Journal of Computer Vision, 62(1), 17-34. https://doi.org/10.1023/B:VISI.0000046587.42611.2c

Raymond, B., Guennebaud, G., \& Barla, P. (2016). Multi-scale rendering of scratched materials using a structured SV-BRDF model. ACM Transactions on Graphics, 35(4). https://doi.org/10.1145/2897824.2925945

Risser, E. (2020). Optimal textures: Fast and robust texture synthesis and style transfer through optimal transport. ArXiv.

Ritchie, J. B., \& van Buren, B. (2020). When scenes look like materials: René Magritte's reversible figure-ground motif. Art \& Perception, 8(3-4), 299-310. https://doi.org/10.1163/22134913-bja10002

Sawayama, M., Nishida, S., \& Shinya, M. (2017). Human perception of subresolution fineness of dense textures based on image intensity statistics. Journal of Vision, 17(4), 8. https://doi.org/10.1167/17.4.8

Sedgwick, H. A. (2005). Visual space perception. In E. B. Goldstein, G. Humphreys, M. 
Shiffrar, \& W. Yost (Eds.), Blackwell handbook of sensation and perception (pp. 138139). Blackwell Publishing.

Sharan, L., Liu, C., Rosenholtz, R., \& Adelson, E. H. (2013). Recognizing materials using perceptually inspired features. International Journal of Computer Vision, 103(3), 348371. https://doi.org/10.1007/s11263-013-0609-0

Sharan, L., Rosenholtz, R., \& Adelson, E. H. (2014). Accuracy and speed of material categorization in real-world images. Journal of Vision, 14(9), 12. Retrieved from http://dx.doi.org/10.1167/14.9.12

Vishwanath, D. (2010). Visual information in surface and depth perception: Reconciling pictures and reality. In L. Albertazzi, G. van Tonder, \& D. Vishwanath (Eds.), Perception beyond inference: The information content of visual processes (pp. 201240). Cambridge, MA: MIT Press. https://doi.org/10.7551/mitpress/8594.003.0012

Wiebel, C. B., Valsecchi, M., \& Gegenfurtner, K. R. (2013). The speed and accuracy of material recognition in natural images. Attention, Perception, \& Psychophysics, 75(5), 954-966. https://doi.org/10.3758/s13414-013-0436-y 